\title{
Irregularity and Density of Irregular Intuitionistic Fuzzy Graphs
}

\author{
${ }^{1} \mathrm{~S}$. Yahya Mohamed and ${ }^{2} \mathrm{R} . J a h i r H u s s a i n$ \\ ${ }^{I} P . G$ and Research Department of Mathematics, Govt. Arts College, Tiruchirappalli-620 022, India \\ ${ }^{2} P . G$ and Research Department of Mathematics, Jamal Mohamed College,Tiruchirappalli-620 020, India
}

\begin{abstract}
In this paper we introduce the notion of Irregularity and total Irregularity of any Irregular Intuitionistic Fuzzy graphs and some results are present. Also Density, Balanced Irregular Intuitionistic Fuzzy graphs are discussed and some of their properties are established.
\end{abstract}

Keywords: Intuitionistic fuzzy graph, degree, Irregularity, Density of IIFG, Balanced IIFG.

\section{Introduction}

Albertson[1] defined measure of Irregularity of Crisp graph and discussed some properties. Atanassov [3] introduced the concept of intuitionistic fuzzy (IF) relations and intuitionistic fuzzy graphs (IFGs). Research on the theory of intuitionistic fuzzy sets (IFSs) has been witnessing an exponential growth in Mathematics and its applications. This ranges from traditional Mathematics to Information Sciences. This leads to consider IFGs and their applications. R. Parvathy and M.G.Karunambigai's paper [4] introduced the concept of IFG and analyzed its components. NagoorGani, A and Sajitha Begum, S [6] defined degree, Order and Size in intuitionistic fuzzy graphs and extend the properties. A. NagoorGani, et. al[13] defined types of Irregular Intuitionistic fuzzy graphs and discussed some properties.

In this paper, we define Irregularity and total Irregularity of any Irregular Intuitionistic fuzzy graphs and some results are attempted. Also Density of Irregular Intuitionistic fuzzy graphs and balanced Irregular Intuitionistic fuzzy graphs are discussed. Some of their properties are established and this concept is useful in Signal processing, artificial intelligence and computer networks.

\section{Preliminaries}

Definition 2.1: An Intuitionistic fuzzy graph is of the form $\mathrm{G}=(\mathrm{V}, \mathrm{E})$ where

(i) $\mathrm{V}=\left\{\mathrm{v}_{1}, \mathrm{v}_{2}, \ldots, \mathrm{V}_{\mathrm{n}}\right\}$ such that $\mu_{1}: \mathrm{V} \rightarrow[0,1]$ and $\gamma_{1}: \mathrm{V} \rightarrow[0,1]$ denote the degree ofmembership and nonmembership of the element vi $€ V$, respectively, and $0 \leq \mu_{1}$ (vi) $+\gamma_{1}(v i) \leq 1$ for every vi $\epsilon V$, (i $\left.=1,2, \ldots \ldots . n\right)$,

(ii) $\mathrm{E} \subseteq \mathrm{V} x \mathrm{~V}$ where $\mu_{2}: \mathrm{VxV} \rightarrow[0,1]$ and $\gamma_{2}: \mathrm{VxV} \rightarrow[0,1]$ are such that $\mu_{2}(\mathrm{vi}, \mathrm{vj}) \leq \min \left[\mu_{1}(\mathrm{vi}), \mu_{1}(\mathrm{vj})\right]$ and $\gamma_{2}(\mathrm{vi}, \mathrm{vj}) \leq \max \left[\gamma_{1}(\mathrm{vi}), \gamma_{1}(\mathrm{vj})\right]$ and $0 \leq \mu_{2}(\mathrm{vi}, \mathrm{vj})+\gamma_{2}(\mathrm{vi}, \mathrm{vj}) \leq 1$ for every $(\mathrm{vi}, \mathrm{vj}) \in \mathrm{E},(\mathrm{i}, \mathrm{j}=1,2, \ldots \ldots \mathrm{n})$

Definition 2.2: An IFG H = ( V', E') is said to be an Intuitionistic fuzzy subgraph (IFSG) of the IFG $\mathrm{G}=(\mathrm{V}, \mathrm{E})$, if $\mathrm{V}^{\prime} \subseteq \mathrm{V}$ and $\mathrm{E}^{\prime} \subseteq \mathrm{E}$. In other words, if $\mu_{1 \mathrm{i}}{ }^{\prime} \leq \mu_{1 \mathrm{i}} ; \gamma_{1 \mathrm{i}}{ }^{\prime} \geq \gamma_{1 \mathrm{i}}$ and $\mu_{2 \mathrm{ij}}{ }^{\prime} \leq \mu_{2 \mathrm{ij}} ; \gamma_{2 \mathrm{ij}}{ }^{\prime} \geq \gamma_{2 \mathrm{ij}}$ for every $\mathrm{i}, \mathrm{j}=1,2 \ldots \ldots \ldots . \mathrm{n}$.

Definition 2.3: An Intuitionistic fuzzy graph is complete if $\mu_{2 \mathrm{ij}}=\min \left(\mu_{1 \mathrm{i}}, \mu_{1 \mathrm{j}}\right)$ and $\gamma_{2 \mathrm{ij}}=\max \left(\gamma_{2 \mathrm{i}}, \gamma_{2 \mathrm{j}}\right)$ for all ( vi , vj ) $€ \mathrm{~V}$.

Definition 2.4:Let $G=(V, E)$ be an IFG. Then the degree of a vertex $v$ is defined by $d(v)=(d \mu(v), d \gamma(v))$ where $\mathrm{d} \mu(\mathrm{v})=\Sigma \mathrm{u} \neq \mathrm{v} \mu_{2}(\mathrm{v}, \mathrm{u})$ and $\mathrm{d} \gamma(\mathrm{v})=\Sigma \mathrm{u} \neq \mathrm{v} \gamma_{2}(\mathrm{v}, \mathrm{u})$.

Definition 2.5: The total degree of a vertex ' $v$ ' is defined as $t(v)=\left(t_{\mu}(v), t_{\gamma}(v)\right)$, where $\mathrm{t}_{\mu}(\mathrm{v})=\Sigma \mathrm{u} \neq \mathrm{v} \mu_{2}(\mathrm{v}, \mathrm{u})+\mu_{1}(\mathrm{v})$ and $\mathrm{t}_{\gamma}(\mathrm{v})=\Sigma \mathrm{u} \neq \mathrm{v} \gamma_{2}(\mathrm{v}, \mathrm{u})+\gamma_{1}(\mathrm{v})$

Definition 2.6: The complement of an IFG G $=(V, E)$ is denoted by $\bar{G}=(\bar{V}, \bar{E})$ and is defined as i) $\bar{\mu}_{1}(\mathrm{v})=\mu_{1}(\mathrm{v})$ and $\bar{\gamma}_{1}(\mathrm{v})=\gamma_{1}(\mathrm{v})$

ii) $\bar{\mu}_{2}(\mathrm{u}, \mathrm{v})=\mu_{1}(\mathrm{u}) \Lambda \mu_{1}(\mathrm{v})-\mu_{2}(\mathrm{u}, \mathrm{v})$ and $\bar{\gamma}_{2}(\mathrm{u}, \mathrm{v})=\gamma_{1}(\mathrm{u}) \mathrm{V} \gamma_{1}(\mathrm{v})-\gamma_{2}(\mathrm{u}, \mathrm{v})$ for $\mathrm{u}, \mathrm{v}$ in $\mathrm{V}$.

Definition 2.7: An Intuitionistic fuzzy graph $G=(V, E)$ is said to be regular, if every vertex has same degree.

Definition 2.8: Let $G=(V, E)$ be IFG. Then $G$ is irregular, if there is a vertex which is adjacent to vertices with distinct degrees.

Definition 2.9: $L e t ~ G=(V, E)$ be a IFG. Then $G$ is totally irregular, if there is a vertex which is adjacent to vertices with distinct total degrees.

Definition 2.10: $L e t ~ G=(V, E)$ be a connected IFG. Then, $G$ is said to be a highly irregular IFG if every vertex of $\mathrm{G}$ is adjacent to vertices with distinct degrees 


\section{Definition2.11:}

A homomorphism of Intuitionistic fuzzy graphs $h: G \rightarrow G$ ' is a map $h: S \rightarrow S^{\prime}$ which satisfies

(i) $\quad \mu_{1}(\mathrm{x}) \leq \mu_{1}^{\prime}(\mathrm{h}(\mathrm{x})) ; \gamma_{1}(\mathrm{x}) \geq \gamma_{1}{ }^{\prime}(\mathrm{h}(\mathrm{x}))$ for every $\mathrm{x} \in \mathrm{S}$.

(ii) $\quad \mu_{2}(x, y) \leq \mu_{2}{ }^{\prime}(h(x), h(y)) ; \gamma_{2}(x, y) \geq \gamma_{2}{ }^{\prime}(h(x), h(y))$ for every $x \in S$.

Definition 2.12:

A weak isomorphism of Intuitionistic fuzzy graphs $h: G \rightarrow G$ ' is a map $h: S \rightarrow S$ ' which is a bijective homomorphism that satisfies $\left(\mu_{1}(\mathrm{x}), \gamma_{1}(\mathrm{x})\right)=\left(\mu_{1}{ }^{\prime}(\mathrm{h}(\mathrm{x})), \gamma_{1}{ }^{\prime}(\mathrm{h}(\mathrm{x}))\right.$ for every $\mathrm{x} \in \mathrm{S}$.

Definition 2.13:

A co-weak isomorphism of intuitionistic fuzzy graphs $h: G \rightarrow G$ ' is a map h: $S \rightarrow S^{\prime}$ which is a bijective homomorphism that satisfies $\left.\mu_{2}(\mathrm{x}, \mathrm{y}), \gamma_{2}(\mathrm{x}, \mathrm{y})\right)=\left(\mu_{2}{ }^{\prime}(\mathrm{h}(\mathrm{x}), \mathrm{h}(\mathrm{y})), \gamma_{2}{ }^{\prime}(\mathrm{h}(\mathrm{x}), \mathrm{h}(\mathrm{y}))\right)$ for every $\mathrm{x}, \mathrm{y} \in \mathrm{S}$.

\section{Definition 2.14:}

An isomorphism of intuitionistic fuzzy graphs $h: G \rightarrow G^{\prime}$ is a map $h: S \rightarrow S^{\prime}$ which is a bijective homomorphism that satisfies

(i) $\quad\left(\mu_{1}(\mathrm{x}), \gamma_{1}(\mathrm{x})\right)=\left(\mu_{1}^{\prime}(\mathrm{h}(\mathrm{x})), \gamma_{1}{ }^{\prime}(\mathrm{h}(\mathrm{x}))\right.$ for every $\mathrm{x} C \mathrm{~S}$.

(ii) $\quad\left(\mu_{2}(\mathrm{x}, \mathrm{y}), \gamma_{2}(\mathrm{x}, \mathrm{y})\right)=\left(\mu_{2}^{\prime}(\mathrm{h}(\mathrm{x}), \mathrm{h}(\mathrm{y})), \gamma_{2}{ }^{\prime}(\mathrm{h}(\mathrm{x}), \mathrm{h}(\mathrm{y}))\right)$ for every $\mathrm{x}, \mathrm{y} \in \mathrm{S}$.

This will denote as $\mathrm{G} \cong \mathrm{G}^{\prime}$.

An IntuitonisticFuzzy graph $\mathrm{G}$ is said to be self complementary if $\mathrm{G} \cong \overline{\mathrm{G}}$.

\section{Irregularity of Intuitionistic Fuzzy Graphs}

Definition 3.1: Let $\mathrm{G}=(\sigma, \mu)$ be any Irregular fuzzy graph then the Irregularity of $\mathrm{G}$ is defined as $\operatorname{Irreg}(\mathrm{G})=\sum_{\mathrm{xy} \in \mathrm{E}}|\mathrm{d}(\mathrm{x})-\mathrm{d}(\mathrm{y})|$ for all $\mathrm{x}, \mathrm{y} \in \mathrm{V}$.

The total Irregularity of the Irregular fuzzy graph is defined as $\operatorname{Irreg}_{t}(G)=\frac{1}{2} \sum_{x, y \in V}|d(x)-d(y)|$

Example 3.2: Let $\mathrm{G}=(\sigma, \mu)$ be any Irregular fuzzy graph with $\mathrm{V}=\{\mathrm{u}, \mathrm{v}, \mathrm{w}, \mathrm{x}\}$ defined by $\sigma(\mathrm{u})=0.6$, $\sigma(\mathrm{v})=0.5, \sigma(\mathrm{w})=0.8, \sigma(\mathrm{x})=1$ and $\mu(\mathrm{u}, \mathrm{v})=0.4, \mu(\mathrm{u}, \mathrm{x})=0.6, \mu(\mathrm{v}, \mathrm{w})=0.3, \mu(\mathrm{w}, \mathrm{x})=0.5$.

Then, $\mathrm{d}(\mathrm{u})=1.0, \mathrm{~d}(\mathrm{v})=0.7, \mathrm{~d}(\mathrm{w})=0.8, \mathrm{~d}(\mathrm{x})=1.1$ and $\operatorname{Irreg}(\mathrm{G})=0.3+0.1+0.3+0.1=0.8$ and $\operatorname{Irreg}_{\mathrm{t}}(\mathrm{G})=\frac{1}{2}[0.3+0.2+0.1+0.1+0.4+0.3]=\frac{1.4}{2}=0.7$

Definition 3.3: Let $G=(V, E)$ be a any type of Irregular Intuitionistic fuzzy graph then the Irregularity of

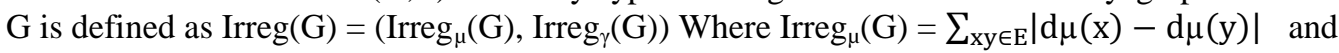
$\operatorname{Irreg}_{\gamma}(\mathrm{G})=\sum_{\mathrm{xy} \in \mathrm{E}}|\mathrm{d} \gamma(\mathrm{x})-\mathrm{d} \gamma(\mathrm{y})|$ for all $\mathrm{x}, \mathrm{y} \in \mathrm{V}$

The total Irregularity of the Irregular Intuitionistic fuzzy graph is defined as

$\operatorname{Irreg}_{\mathrm{t}}(\mathrm{G})=\frac{1}{2} \sum_{\mathrm{x}, \mathrm{y} \in \mathrm{V}}|\mathrm{d}(\mathrm{x})-\mathrm{d}(\mathrm{y})|$

Example 3.4:

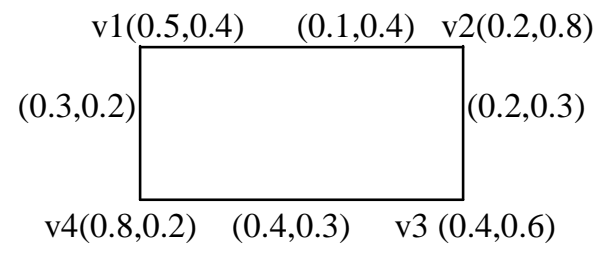

Fig -1: Irregular Intuitionistic fuzzy graph

Here $\mathrm{d}(\mathrm{v} 1)=(0.4,0.6), \mathrm{d}(\mathrm{v} 2)=(0.3,0.7), \mathrm{d}(\mathrm{v} 3)=(0.6,0.6)$ and $\mathrm{d}(\mathrm{v} 4)=(0.7,0.5)$.

Then, $\operatorname{Irreg}(\mathrm{G})=(0.1,0.1)+(0.3,0.1)+(0.1,0.1)+(0.3,0.1)=(0.8,0.4)$.

But the total Irregularity is $\operatorname{Irreg}_{\mathrm{t}}(\mathrm{G})=\frac{1}{2}[(0.1,0.1)+(0.2,0.0)+(0.3,0.1)+(0.3,0.1)+(0.4,0.2)+(0.1,0.1)$

\section{Remark 3.5 :}

$$
=(0.7,0.3)
$$

(1) If $G$ is regular Intuitionistic fuzzy graph then $\operatorname{Irreg}(G)=0$

(2) For any Irregular Intuitionistic fuzzy graph $\operatorname{Irreg}_{t}(G) \leq \operatorname{Irreg}(G)$

(3) Let $G=(V, E)$ be the Irregular Intuitionistic fuzzy graph which is complete then $\operatorname{Irreg}(G)=2\left[\operatorname{Irreg}_{t}(G)\right]$ 
Theorem 3.6: Let $G=(V, E)$ be the Irregular IFG with all vertices having edges with $\mu_{2}(x, y)=1 / 2\left(\mu_{1}(x) \Lambda \mu_{1}\right.$ $(\mathrm{y}))$ and $\gamma_{2}(\mathrm{x}, \mathrm{y})=1 / 2\left(\gamma_{1}(\mathrm{x}) \vee \gamma_{1}(\mathrm{y})\right)$ and $\mu_{1}+\gamma_{1}=1$ then $\operatorname{Irreg}_{\mu}(\mathrm{G})=\operatorname{Irreg}_{\mathrm{\gamma}}(\mathrm{G})$ for all edges.

\section{Example 3.7:}

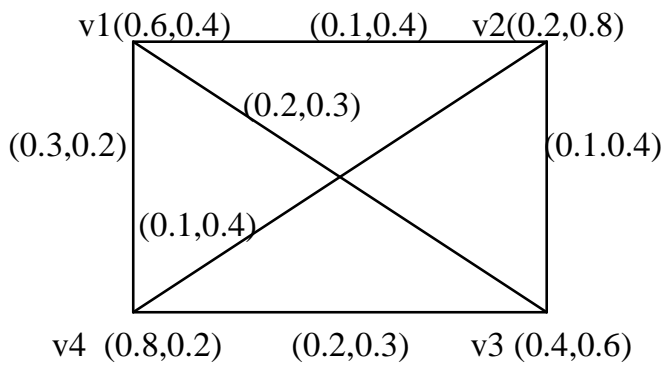

Fig -2:Irregualr but not highly Irregular IFG

Here $\mathrm{d}(\mathrm{v} 1)=(0.6,0.9), \mathrm{d}(\mathrm{v} 2)=(0.3,1.2), \mathrm{d}(\mathrm{v} 3)=(0.5,1.0)$ and $\mathrm{d}(\mathrm{v} 4)=(0.6,0.9)$.

So Irreg $(\mathrm{G})=(0.3,0.3)+(0.2,0.2)+(0.1,0.1)+(0,0)+(0.3,0.3)+(0.1,0.1)=(1.0,1.0)$.

i.e) $\operatorname{Irreg}_{\mu}(G)=\operatorname{Irreg}_{\gamma}(G)$

Theorem3.8: Let $\mathrm{G}=(\mathrm{V}, \mathrm{E})$ be the Irregular IFG with $\mu_{2}(\mathrm{vi}, \mathrm{vj})+\gamma_{2}(\mathrm{vi}, \mathrm{vj})$ is same for every $(\mathrm{vi}, \mathrm{vj}) \in \mathrm{E}$, $(i, j=1,2, \ldots \ldots . n)$ then $\operatorname{Irreg}_{\mu}(G)=\operatorname{Irreg}_{\gamma}(G)$.

\section{Example 3.7:}

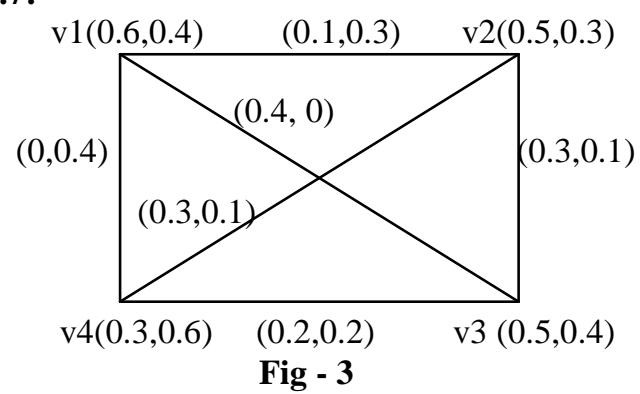

Here $\mathrm{d}(\mathrm{v} 1)=(0.5,0.7), \mathrm{d}(\mathrm{v} 2)=(0.7,0.5), \mathrm{d}(\mathrm{v} 3)=(0.9,0.3)$ and $\mathrm{d}(\mathrm{v} 4)=(0.5,0.7)$. So Irreg $(\mathrm{G})=(0.2,0.2)+$ $(0.2,0.2)+(0.4,0.4)+(0.4,0.4)+((0.0,0.0)+(0.2,0.2)=(1.4,1.4)$.

i.e) $\operatorname{Irreg}_{\mu}(\mathrm{G})=\operatorname{Irreg}_{\gamma}(\mathrm{G})$.

Theorem 3.9:Let $G=(V, E)$ be the Irregular IFG with $d \mu(V i)+d \gamma(V i)=d \mu(V j)+d \gamma(V j)$ for all $V i, V j \in V$ then $\operatorname{Irreg}_{\mu}(G)=\operatorname{Irreg}_{\gamma}(G)$.

Proof:Given $d \mu(V i)+d \gamma(V i)=d \mu(V j)+d \gamma(V j)$ then $\mathrm{d} \mu(\mathrm{Vi})-\mathrm{d} \mu(\mathrm{Vj})=\mathrm{d} \gamma(\mathrm{Vi})-\mathrm{d} \gamma(\mathrm{Vj})$ for all $\mathrm{Vi}, \mathrm{Vj} \in \mathrm{V}$

$\sum_{\mathrm{ViVj}}|\mathrm{d} \mu(\mathrm{Vi})-\mathrm{d} \mu(\mathrm{Vj})|=\sum_{\mathrm{ViVj}}|\mathrm{d} \gamma(\mathrm{Vi})-\mathrm{d} \gamma(\mathrm{Vj})|$

i.e) $\operatorname{Irreg}_{\mu}(\mathrm{G})=\operatorname{Irreg}_{\gamma}(\mathrm{G})$.

Theorem 3.10 : Let $\mathrm{G}=(\mathrm{V}, \mathrm{E})$ and $\mathrm{G}^{1}=\left(\mathrm{V}^{1}, \mathrm{E}^{1}\right)$ be two Irregular IFG and $\mathrm{G}$ is co-weak isomorphic with $\mathrm{G}^{1}$ then $\operatorname{Irreg}(G)=\operatorname{Irreg}\left(G^{1}\right)$.

Proof: Since $G$ is co-weak isomorphic with $G^{1}$ then $\left(\mu_{2}(x, y), \gamma_{2}(x, y)\right)=\left(\mu_{2}{ }^{\prime}(h(x), h(y)), \gamma_{2}{ }^{\prime}(h(x), h(y))\right)$ for every $\mathrm{x}, \mathrm{y} \in \mathrm{S}$.

Now $\mathrm{d} \mu(\mathrm{x})=\Sigma \mathrm{x} \neq \mathrm{y} \mu_{2}(\mathrm{x}, \mathrm{y})=\Sigma \mathrm{x} \neq \mathrm{y} \mu_{2}{ }^{\prime}((\mathrm{h}(\mathrm{x}), \mathrm{h}(\mathrm{y}))=\mathrm{d} \mu(\mathrm{h}(\mathrm{x})$

Therefore $\sum_{\mathrm{xy} \in \mathrm{E}}|\mathrm{d} \mu(\mathrm{x})-\mathrm{d} \mu(\mathrm{y})|=\sum_{\mathrm{xy} \in \mathrm{E}}|\mathrm{d} \mu(\mathrm{h}(\mathrm{x}))-\mathrm{d} \mu(\mathrm{h}(\mathrm{y}))|$

Similarly $\sum_{\mathrm{xy} \in \mathrm{E}}|\mathrm{d} \gamma(\mathrm{x})-\mathrm{d} \gamma(\mathrm{y})| \quad=\sum_{\mathrm{xy} \in \mathrm{E}}|\mathrm{d} \gamma(\mathrm{h}(\mathrm{x}))-\mathrm{d} \gamma(\mathrm{h}(\mathrm{y}))| \quad$ for every $\mathrm{x}, \mathrm{y} \in \mathrm{S}$.

i.e) Irreg $(\mathrm{G})=\operatorname{Irreg}\left(\mathrm{G}^{1}\right)$

\section{Density of Irregular Intuitionistic Fuzzy Graphs}

Definition 4.1: Let $G=(V, E)$ be a Irregular IFG. The Density of $G$ is defined as $D(G)=\left(D_{\mu}(G), D_{\gamma}(G)\right)$ Where $D_{\mu}(G)=\frac{2 \sum_{x, y \in V}\left(\mu_{2}(x, y)\right)}{\sum_{x y \in E}\left(\mu_{1}(x) \Lambda \mu_{1}(y)\right)}$ for all $x, y \in \operatorname{Vand} D_{\gamma}(G)=\frac{2 \sum_{x, y \in V}\left(\gamma_{2}(x, y)\right)}{\sum_{x y \in E}\left(\gamma_{1}(x) V \gamma_{1}(y)\right)} \quad$ for all $x, y \in V$ 
Example 4.2:

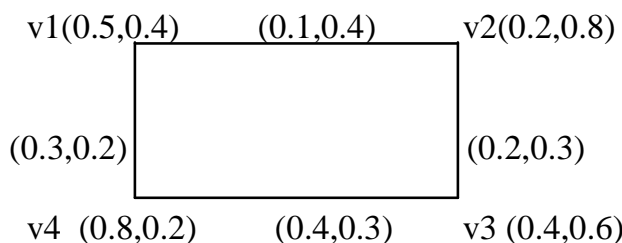

Fig - 4: Highly, neighbourly and totally Irregular IFG.

$\mathrm{D}(\mathrm{G})=\left(\mathrm{D}_{\mu}(\mathrm{G}), \mathrm{D}_{\gamma}(\mathrm{G})\right)=\left(\frac{2[0.1+0.2+0.4+0.3]}{0.2+0.2+0.4+0.5}, \frac{2[0.4+0.3+0.3+0.2]}{0.8+0.8+0.6+0.4}\right)=(1.54,0.923)$

Theorem 4.3: Let $\mathrm{G}=(\mathrm{V}, \mathrm{E})$ be the Irregular Intuitionistic fuzzy graph with $\mu_{2}(\mathrm{x}, \mathrm{y})=1 / 2\left(\mu_{1}(\mathrm{x}) \Lambda \mu_{1}(\mathrm{y})\right)$ and $\gamma_{2}$ $(\mathrm{x}, \mathrm{y})=1 / 2\left(\gamma_{1}(\mathrm{x}) \mathrm{V} \gamma_{1}(\mathrm{y})\right)$ then $\mathrm{D}(\mathrm{G})=(1,1)$

Proof:

$$
\begin{aligned}
\mathrm{D}(\mathrm{G})= & \left(\frac{2 \sum_{\mathrm{x}, \mathrm{y} \in \mathrm{V}}\left(\mu_{2}(\mathrm{x}, \mathrm{y})\right)}{\sum_{\mathrm{xy} \in \mathrm{E}}\left(\mu_{1}(\mathrm{x}) \Lambda \mu_{1}(\mathrm{y})\right)}, \frac{2 \sum_{\mathrm{x}, \mathrm{y} \in \mathrm{V}}\left(\gamma_{2}(\mathrm{x}, \mathrm{y})\right)}{\sum_{\mathrm{xy} \in \mathrm{E}}\left(\gamma_{1}(\mathrm{x}) \mathrm{V} \gamma_{1}(\mathrm{y})\right)}\right) \quad \text { for all } \mathrm{x}, \mathrm{y} \in \mathrm{V} \\
= & \left(\frac{2 \sum_{\mathrm{x}, \mathrm{y} \in \mathrm{V}} \frac{1}{2}\left(\mu_{1}(\mathrm{x}) \Lambda \mu_{1}(\mathrm{y})\right)}{\sum_{\mathrm{xy} \in \mathrm{E}}\left(\mu_{1}(\mathrm{x}) \Lambda \mu_{1}(\mathrm{y})\right)}, \frac{2 \sum_{\mathrm{x}, \mathrm{y} \in \mathrm{V}} \frac{1}{2}\left(\gamma_{1}(\mathrm{x}) \mathrm{V} \gamma_{1}(\mathrm{y})\right)}{\sum_{\mathrm{xy} \in \mathrm{E}}\left(\gamma_{1}(\mathrm{x}) \mathrm{V} \gamma_{1}(\mathrm{y})\right)}\right) \\
= & \left(\frac{2\left(\frac{1}{2}\right) \sum_{\mathrm{x}, \mathrm{y} \in \mathrm{V}}\left(\mu_{1}(\mathrm{x}) \Lambda \mu_{1}(\mathrm{y})\right)}{\sum_{\mathrm{xy} \in \mathrm{E}}\left(\mu_{1}(\mathrm{x}) \Lambda \mu_{1}(\mathrm{y})\right)}, \frac{2\left(\frac{1}{2}\right) \sum_{\mathrm{x}, \mathrm{y} \in \mathrm{V}}\left(\gamma_{1}(\mathrm{x}) \mathrm{V} \gamma_{1}(\mathrm{y})\right)}{\sum_{\mathrm{xy} \in \mathrm{E}}\left(\gamma_{1}(\mathrm{x}) \mathrm{V} \gamma_{1}(\mathrm{y})\right)}\right)=(1,1)
\end{aligned}
$$

Theorem 4.4: Let $G=(V, E)$ be Irregular and complete IFG then $D(G)=(2,2)$

Proof:

Given $\mathrm{G}$ is Irregular and complete IFG then

$\mu_{2}(\mathrm{x}, \mathrm{y})=\mu_{1}(\mathrm{x}) \Lambda \mu_{1}(\mathrm{y})$ and $\gamma_{2}(\mathrm{x}, \mathrm{y})=\gamma_{1}(\mathrm{x}) \mathrm{V} \gamma_{1}(\mathrm{y})$ for all $\mathrm{x}, \mathrm{y} \in \mathrm{V}$

Now $\sum_{\mathrm{x}, \mathrm{y} \in \mathrm{V}}\left(\mu_{2}(\mathrm{x}, \mathrm{y})\right)=\sum_{\mathrm{xy} \in \mathrm{E}}\left(\mu_{1}(\mathrm{x}) \Lambda \mu_{1}(\mathrm{y})\right)$, Also $\sum_{\mathrm{x}, \mathrm{y} \in \mathrm{V}}\left(\gamma_{2}(\mathrm{x}, \mathrm{y})\right)=\sum_{\mathrm{xy} \in \mathrm{E}}\left(\gamma_{1}(\mathrm{x}) \operatorname{V} \gamma_{1}(\mathrm{y})\right)$

Therefore $D(G)=\left(\frac{2 \sum_{x, y \in V}\left(\mu_{2}(x, y)\right)}{\sum_{x y \in E}\left(\mu_{1}(x) \Lambda \mu_{1}(y)\right)}, \frac{2 \sum_{x, y \in V}\left(\gamma_{2}(x, y)\right)}{\sum_{x y \in E}\left(\gamma_{1}(x) V \gamma_{1}(y)\right)}\right) \quad$ for all $x, y \in V$

$=\left(\frac{2 \sum_{\mathrm{xy} \in \mathrm{E}}\left(\mu_{1}(\mathrm{x}) \Lambda \mu_{1}(\mathrm{y})\right)}{\sum_{\mathrm{xy} \in \mathrm{E}}\left(\mu_{1}(\mathrm{x}) \Lambda \mu_{1}(\mathrm{y})\right)}, \frac{2 \sum_{\mathrm{xy} \in \mathrm{E}}\left(\gamma_{1}(\mathrm{x}) \mathrm{V} \gamma_{1}(\mathrm{y})\right)}{\sum_{\mathrm{xy} \in \mathrm{E}}\left(\gamma_{1}(\mathrm{x}) \mathrm{V} \gamma_{1}(\mathrm{y})\right)}\right)$ for all $\mathrm{x}, \mathrm{y} \in \mathrm{V}$

$$
=(2,2)
$$

Definition4.5: An Irregular IFG $G$ is said to be balanced if $D_{\mu}(H) \leq D_{\mu}(G)$ and $D_{\gamma}(H) \leq D_{\gamma}(G)$ for all subgraphs $\mathrm{H}$ of $\mathrm{G}$.

\section{Example 4.6}

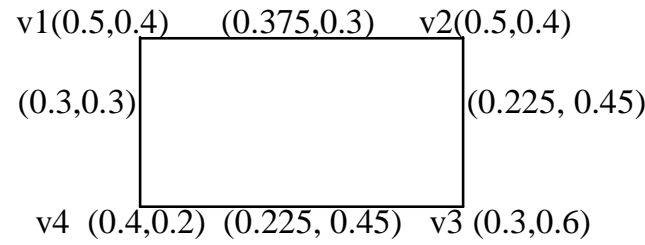

Fig - 5: Balanced Irregular IFG (G)

Here $\mathrm{D}(\mathrm{G})=\left(\mathrm{D}_{\mu}(\mathrm{G}), \mathrm{D}_{\gamma}(\mathrm{G})\right)=(1.5,1.5)$ and sub graphs of $\mathrm{G}$ are

$\mathrm{H} 1=\{\mathrm{v} 1, \mathrm{v} 2\}, \mathrm{H} 2=\{\mathrm{v} 1, \mathrm{v} 3\}, \mathrm{H} 3=\{\mathrm{v} 1, \mathrm{v} 4\}, \mathrm{H} 4=\{\mathrm{v} 2, \mathrm{v} 3\}, \mathrm{H} 5=\{\mathrm{v} 2, \mathrm{v} 4\}, \mathrm{H} 6=\{\mathrm{v} 3, \mathrm{v} 4, \mathrm{H} 7=\{\mathrm{v} 1, \mathrm{v} 2, \mathrm{v} 3\}$,

$\mathrm{H} 8=\{\mathrm{v} 1, \mathrm{v} 3, \mathrm{v} 4\}, \mathrm{H} 9=\{\mathrm{v} 1, \mathrm{v} 2, \mathrm{v} 4\}, \mathrm{H} 10=\{\mathrm{v} 2, \mathrm{v} 3, \mathrm{v} 4\}, \mathrm{H} 11=\{\mathrm{v} 1, \mathrm{v} 2, \mathrm{v} 3, \mathrm{v} 4\}$.

Now Density $(\mathrm{D} \mu(\mathrm{H}), \mathrm{D} v(\mathrm{H}))$ is $\mathrm{D}(\mathrm{H} 1)=(1.5,1.5), \mathrm{D}(\mathrm{H} 2)=(0,0), \mathrm{D}(\mathrm{H} 3)=(1.5,1.5), \mathrm{D}(\mathrm{H} 4)=(1.5,1.5)$,

$\mathrm{D}(\mathrm{H} 5)=(0,0), \mathrm{D}(\mathrm{H} 6)=(15,1.5), \mathrm{D}(\mathrm{H} 7)=(1.5,1.5), \mathrm{D}(\mathrm{H} 8)=(1.5,1.5), \mathrm{D}(\mathrm{H} 9)=(1.5,1.5), \mathrm{D}(\mathrm{H} 10)=(15,1.5)$,

$\mathrm{D}(\mathrm{H} 11)=(1.5,1.5)$.

So $\mathrm{D}(\mathrm{H}) \leq \mathrm{D}(\mathrm{G})$ for all subgraphs $\mathrm{H}$ of $\mathrm{G}$. Hence $\mathrm{G}$ is balanced Irregular IFG.

Definition 4.7: An Irregular IFG G is said to be strictly balanced Irregular IFG $\operatorname{ifD}_{\mu}(H)=D_{\mu}(G)$ and $D_{\gamma}(H)=$ $\mathrm{D}_{\gamma}(\mathrm{G})$ for all non emptysubgraphs $\mathrm{H}$ of G.i.e) $\mathrm{D}(\mathrm{H})=\mathrm{D}(\mathrm{G})$ 


\section{Example 4.8:}

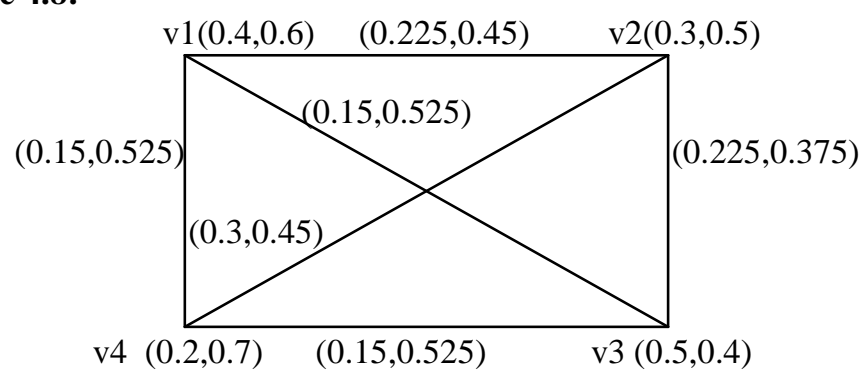

Fig - 6: Strictly Balanced Irregular IFG

In this Graph $\mathrm{D}(\mathrm{G})=\left(\frac{2[0.225+0.225+0.15+0.15+0.15+0.3]}{03+0.3+0.2+0.2+0.2+0.4}, \frac{2[0.45+0.375+0.525+0.525+0.525+0.45]}{0.6+0.5+0.7+0.7+0.6+0.7}\right)=(1.5,1.5)$

And for all the non-empty subgraphs $\mathrm{H}$ of $\mathrm{G}, \mathrm{D}(\mathrm{H})=(1.5,1.5)$.

i.e) $\mathrm{D}(\mathrm{H})=\mathrm{D}(\mathrm{G})$. Therefore $\mathrm{G}$ is Strictly Balanced Irregular IFG.

Theorem 4.9: Let $G=(V, E)$ be strictly balancedtotally Irregular IFG then $D(G)+D(\bar{G})=(2,2)$ and the complement also totally Irregular IFG.

Proof: Let $G$ be the totally Irregular IFG. Also $G$ is Strictly balanced $\rightarrow D(H)=D(G)$

Let $\overline{\mathrm{G}}=(\overline{\mathrm{V}}, \overline{\mathrm{E}})$ be complement of $\mathrm{G}$ then

(i) $\bar{\mu}_{1}(\mathrm{x})=\mu_{1}(\mathrm{x})$ and $\bar{\gamma}_{1}(\mathrm{x})=\gamma_{1}(\mathrm{x})$ for all $\mathrm{x}$ in $\mathrm{V}$ and (ii) $\bar{\mu}_{2}(\mathrm{x}, \mathrm{y})=\mu_{1}(\mathrm{x}) \Lambda \mu_{1}(\mathrm{y})-\mu_{2}(\mathrm{x}, \mathrm{y}) \rightarrow(1)$

and $\bar{\gamma}_{2}(\mathrm{x}, \mathrm{y})=\gamma_{1}(\mathrm{x}) \mathrm{V} \gamma_{1}(\mathrm{y})-\gamma_{2}(\mathrm{x}, \mathrm{y})$ for $\mathrm{x}, \mathrm{y}$ in $\mathrm{V} \rightarrow(2)$

Dividing (1) by $\mu_{1}$ (x) $\Lambda \mu_{1}$ (y) on both sides

$$
\frac{\bar{\mu}_{2}(\mathrm{x}, \mathrm{y})}{\mu 1(\mathrm{x}) \Lambda \mu 1(\mathrm{y})}=1-\frac{\mu_{2}(\mathrm{x}, \mathrm{y})}{\mu 1(\mathrm{x}) \Lambda \mu 1(\mathrm{y})}
$$

Then $\sum_{\mathrm{x}, \mathrm{y} \in \mathrm{V}} \frac{\bar{\mu}_{2}(\mathrm{x}, \mathrm{y})}{\mu 1(\mathrm{x}) \Lambda \mu 1(\mathrm{y})}=1-\sum_{\mathrm{x}, \mathrm{y} \in \mathrm{V}} \frac{\left.\mu_{2}(\mathrm{x}, \mathrm{y})\right)}{\mu 1(\mathrm{x}) \Lambda \mu 1(\mathrm{y})}$

$2 \sum_{\mathrm{x}, \mathrm{y} \in \mathrm{V}} \frac{\bar{\mu}_{2}(\mathrm{x}, \mathrm{y})}{\mu 1(\mathrm{x}) \Lambda \mu 1(\mathrm{y})}=2-2 \sum_{\mathrm{x}, \mathrm{y} \in \mathrm{V}} \frac{\left.\mu_{2}(\mathrm{x}, \mathrm{y})\right)}{\mu 1(\mathrm{x}) \Lambda \mu 1(\mathrm{y})} \quad$ for all $\mathrm{x}, \mathrm{y} \in \mathrm{V}$

$2 \sum_{\mathrm{x}, \mathrm{y} \in \mathrm{V}} \frac{\bar{\mu}_{2}(\mathrm{x}, \mathrm{y})}{\bar{\mu}_{1}(\mathrm{x}) \Lambda \bar{\mu}_{1}(\mathrm{y})}=2-2 \sum_{\mathrm{x}, \mathrm{y} \in \mathrm{V}} \frac{\left.\mu_{2}(\mathrm{x}, \mathrm{y})\right)}{\mu 1(\mathrm{x}) \Lambda \mu 1(\mathrm{y})} \quad$ by (i)

i.e) $D_{\mu}(\bar{G})=2-D_{\mu}(G)$

Similarly Dividing (2) by $\gamma_{1}(\mathrm{x}) \mathrm{V} \gamma_{1}(\mathrm{y})$ on both sides

$$
\frac{\bar{\gamma}_{2}(\mathrm{x}, \mathrm{y})}{\gamma_{1}(\mathrm{x}) \mathrm{V} \gamma_{1}(\mathrm{y})}=1-\frac{\gamma_{2}(\mathrm{x}, \mathrm{y})}{\gamma_{1}(\mathrm{x}) \mathrm{V} \gamma_{1}(\mathrm{y})}
$$

$2 \sum_{\mathrm{x}, \mathrm{y} \in \mathrm{V}} \frac{\bar{\gamma}_{2}(\mathrm{x}, \mathrm{y})}{\bar{\gamma}_{1}(\mathrm{x}) \mathrm{V} \bar{\gamma}_{1}(\mathrm{y})}=2-2 \sum_{\mathrm{x}, \mathrm{y} \in \mathrm{V}} \frac{\gamma_{2}(\mathrm{x}, \mathrm{y})}{\gamma_{1}(\mathrm{x}) \mathrm{V} \gamma_{1}(\mathrm{y})}$ for all $\mathrm{x}, \mathrm{y} \in \mathrm{V}$

i.e) $D_{\gamma}(\bar{G})=2-D_{\gamma}(G)$

$$
\text { Now, } \begin{aligned}
D(G)+D(\bar{G}) & =\left(D_{\mu}(G), D_{\gamma}(G)\right)+\left(D_{\mu}(\bar{G}), D_{\gamma}(\bar{G})\right) \\
& =\left(D_{\mu}(G)+D_{\mu}(\bar{G}), D_{\gamma}(G)+D_{\gamma}(\bar{G})\right)=\left(D_{\mu}(G)+2-D_{\mu}(G), D_{\gamma}(G)+2-D_{\gamma}(G)\right) \\
& =(2,2)
\end{aligned}
$$

Theorem 4.10: Let $G=(V, E)$ and $G^{1}=\left(V^{1}, E^{1}\right)$ be two isomorphic totally Irregular IFGs . If $G^{1}$ is balanced then $\mathrm{G}$ is balanced. Also the subgraphs are also totally Irregular IFGs.

Proof:Let $h: G \rightarrow G^{\prime}$ is a map $h: S \rightarrow S^{\prime}$ which is a bijective homomorphism that satisfies

(i) $\quad\left(\mu_{1}(\mathrm{x}), \gamma_{1}(\mathrm{x})\right)=\left(\mu_{1}^{\prime}(\mathrm{h}(\mathrm{x})), \gamma_{1}{ }^{\prime}(\mathrm{h}(\mathrm{x}))\right.$ for every $\mathrm{x} \in \mathrm{S}$.

(ii) $\quad\left(\mu_{2}(\mathrm{x}, \mathrm{y}), \gamma_{2}(\mathrm{x}, \mathrm{y})\right)=\left(\mu_{2}^{\prime}(\mathrm{h}(\mathrm{x}), \mathrm{h}(\mathrm{y})), \gamma_{2}{ }^{\prime}(\mathrm{h}(\mathrm{x}), \mathrm{h}(\mathrm{y}))\right)$ for every $\mathrm{x}, \mathrm{yCS}$.

Then $\sum_{\mathrm{x} \in \mathrm{V}} \mu_{1}(\mathrm{x})=\sum_{\mathrm{x} \in \mathrm{V}^{1}} \mu_{1}{ }^{1}(\mathrm{x}) \quad$ and $\sum_{\mathrm{x} \in \mathrm{V}} \gamma_{1}(\mathrm{x})=\sum_{\mathrm{x} \in \mathrm{V}^{1}} \gamma_{1}{ }^{1}(\mathrm{x})$

Also $\sum_{\mathrm{x}, \mathrm{y} \in \mathrm{V}} \mu_{2}(\mathrm{x}, \mathrm{y})=\sum_{\mathrm{x}, \mathrm{y} \in \mathrm{V}^{1}} \mu_{2}{ }^{1}(\mathrm{x}, \mathrm{y})$ and $\sum_{\mathrm{x}, \mathrm{y} \in \mathrm{V}} \gamma_{2}(\mathrm{x}, \mathrm{y})=\sum_{\mathrm{x}, \mathrm{y} \in \mathrm{V}^{1}} \gamma_{2}{ }^{1}(\mathrm{x}, \mathrm{y})$

Let $\mathrm{H}_{2}=\mathrm{G}\left(\mathrm{V}\left(\mathrm{H}_{2}\right), \mathrm{E}\left(\mathrm{H}_{2}\right)\right)$ is an Intuitionistic fuzzy subgraph of $\mathrm{G}^{1}$.

Since $G^{1}$ is balanced then $\mathrm{D} \mu\left(\mathrm{H}_{2}\right) \leq \mathrm{D} \mu\left(\mathrm{G}^{1}\right)$ and $\mathrm{D} \gamma\left(\mathrm{H}_{2}\right) \leq \mathrm{D} \gamma\left(\mathrm{G}^{1}\right)$ 


$$
\begin{aligned}
& \left(\frac{2 \sum_{\mathrm{x}, \mathrm{y} \in \mathrm{V}(\mathrm{H} 1)} \mu_{2}{ }^{1}(\mathrm{~h}(\mathrm{x}), \mathrm{h}(\mathrm{y}))}{\sum_{\mathrm{xy} \in \mathrm{E}(\mathrm{H} 1)}\left(\mu_{1}{ }^{1}(\mathrm{~h}(\mathrm{x})) \Lambda \mu_{1}{ }^{1}(\mathrm{~h}(\mathrm{y}))\right.}, \frac{2 \sum_{\mathrm{x}, \mathrm{y} \in \mathrm{V}(\mathrm{H} 1) \gamma_{2}{ }^{1}(\mathrm{~h}(\mathrm{x}), \mathrm{h}(\mathrm{y}))}}{\sum_{\mathrm{xy} \in \mathrm{E}(\mathrm{H} 1)}\left(\gamma_{1}{ }^{1}(\mathrm{~h}(\mathrm{x})) \mathrm{V}{\gamma_{1}}^{1}(\mathrm{~h}(\mathrm{y}))\right)}\right) \\
& \leq\left(\frac{2 \sum_{\mathrm{x}, \mathrm{y} \in \mathrm{V}^{1} \mu_{2}{ }^{1}(\mathrm{~h}(\mathrm{x}), \mathrm{h}(\mathrm{y}))}}{\sum_{\mathrm{xy} \in \mathrm{E}^{1}}\left(\mu_{1}{ }^{1}(\mathrm{~h}(\mathrm{x})) \Lambda \mu_{1}{ }^{1}(\mathrm{~h}(\mathrm{y}))\right.}, \frac{2 \sum_{\mathrm{x}, \mathrm{y} \in \mathrm{V}^{1} \gamma_{2}{ }^{1}(\mathrm{~h}(\mathrm{x}), \mathrm{h}(\mathrm{y})}}{\left.\sum_{\mathrm{xy} \in \mathrm{E}^{1}\left(\gamma_{1}{ }^{1}(\mathrm{~h}(\mathrm{x})) \mathrm{V} \gamma_{1}{ }^{1}(\mathrm{~h}(\mathrm{y}))\right)}\right)}\right. \\
& \left(\frac{2 \sum_{\mathrm{x}, \mathrm{y} \in \mathrm{V}(\mathrm{H} 1)}\left(\mu_{2}(\mathrm{x}, \mathrm{y})\right)}{\sum_{\mathrm{xy} \in \mathrm{E}(\mathrm{H} 1)}\left(\mu_{1}(\mathrm{x}) \Lambda \mu_{1}(\mathrm{y})\right)}, \frac{2 \sum_{\mathrm{x}, \mathrm{y} \in \mathrm{V}(\mathrm{H} 1)\left(\gamma_{2}(\mathrm{x}, \mathrm{y})\right)}}{\left.\sum_{\mathrm{xy} \in \mathrm{E}(\mathrm{H} 1)}\left(\gamma_{1}(\mathrm{x}) \mathrm{V} \gamma_{1}(\mathrm{y})\right)\right)}\right) \leq\left(\frac{2 \sum_{\mathrm{x}, \mathrm{y} \in \mathrm{V}}\left(\mu_{2}(\mathrm{x}, \mathrm{y})\right)}{\sum_{\mathrm{xy} \in \mathrm{E}}\left(\mu_{1}(\mathrm{x}) \Lambda \mu_{1}(\mathrm{y})\right)}, \frac{2 \sum_{\mathrm{x}, \mathrm{y} \in \mathrm{V}}\left(\gamma_{2}(\mathrm{x}, \mathrm{y})\right)}{\sum_{\mathrm{xy} \in \mathrm{E}}\left(\gamma_{1}(\mathrm{x}) \mathrm{V} \gamma_{1}(\mathrm{y})\right)}\right)
\end{aligned}
$$

i.e) $\left(\mathrm{D} \mu\left(\mathrm{H}_{1}\right), \mathrm{D} \gamma\left(\mathrm{H}_{1}\right)\right) \leq(\mathrm{D} \mu(\mathrm{G}), \mathrm{D} \gamma(\mathrm{G}))$

Hence $\mathrm{G}$ is balanced.

Theorem 4.11: Let $\mathrm{G}=(\mathrm{V}, \mathrm{E})$ be Irregular IFG and all the edges of $\mathrm{G}$ are strong the $\mathrm{G}$ is balanced

Proof: $\quad$ Since all the edges are strong then $\mu_{2}(\mathrm{x}, \mathrm{y})=\mu_{1}(\mathrm{x}) \Lambda \mu_{1}(\mathrm{y})$ and $\gamma_{2}(\mathrm{x}, \mathrm{y})=\gamma_{1}(\mathrm{x}) \mathrm{V} \gamma_{1}(\mathrm{y})$

Now, by theorem $3.4 \mathrm{D}(\mathrm{G})=(2,2)$.

For the subgraphs $H,(D \mu(H), D \gamma(G))=(2,2)$ if the vertices of $H$ having edges,

otherwise $(\mathrm{D} \mu(\mathrm{H}), \mathrm{D} \gamma(\mathrm{G}))=(0,0)$.

i.e) $\mathrm{D} \mu(\mathrm{H}) \leq \mathrm{D} \mu(\mathrm{G})$ Which implies $\mathrm{G}$ is Balanced.

Theorem 4.12: Every Irregular IFG which is complete is balanced

Proof:

Let $\mathrm{G}$ be Irregular IFG and complete then by theorem 3.4, $\mathrm{D}(\mathrm{G})=(2,2)$. Let $\mathrm{H}$ be a non emptysubgraphs of $\mathrm{G}$ and $\mathrm{G}$ is complete then, all the edges are Strong. Therefore, $\mathrm{D}(\mathrm{H})=(2,2)$ for every $\mathrm{H}$ in $\mathrm{G}$. Also the subgraphs are totally Irregular IFGs.

i.e) $\mathrm{G}$ is balanced

Remark: The converse need not be true. That is Every balanced Irregular IFG need not be Complete.

Example: Fig- 5 is Balanced Irregular IFG, But is not complete.

\section{Conclusion}

Here we just define the new concept Irregularity and total Irregularity of Irregular Intuitionistic fuzzy graphs of any type and discussed some results. Also some properties of Density of Irregular Intuitionistic fuzzy graphs are derived. Finally, we studied balanced Irregular Intuitionistic fuzzy graphs and its properties. Further, we going to study more results on Irregularity of all the types of Irregular IFGs.

\section{References}

[1] Albertson,M.O. The Irregularity of a graph, Arscombin. 46(1997), 219-225.

[2] AL-Hawary. T, Complete fuzzy graph, International J. Math. Combinatory 4(2011),26-34

[3] Atanassov. KT. Intuitionistic fuzzy sets: theory and applications. Physica, New York, 1999.

[4] Parvathi, R. and Karunambigai, M.G., Intuitionistic Fuzzy Graphs, Computational Intelligence, Theory and applications, International Conference in Germany, Sept 18 -20, 2006.

[5] A.NagoorGani and S.R. Latha, On Irregular Fuzzy Graphs, Applied Mathematical Sciences,Vol.6, 2012, no.11,517-523

[6] A.NagoorGani and S.ShajithaBegum, Degree,Order and Size in Intuitionistic Fuzzy Graphs,International Journal of Algorithms, Computing and Mathematics,(3)3 (2010).

[7] Bhutani,K.R., On Automorphism of Fuzzy graphs, Pattern Recognition Letter 9:159-162,1989.

[8] NagoorGani, A. and Malarvizhi. J, Isomorphism on Fuzzy graphs, International Journal of Computational and Mathematical Sciences 2;4:200-206,2008.

[9] Rucinski. A and Vince. A, The solution to an extremal problem on balanced extensions of graphs, J. of Graph Theory 17(1993), 417-431.

[10] Sunitha, M.S. and Vijayakumar, A., Complement of a Fuzzy graph, Indian J. Pure appl. Math. 33(9):1451-1464, September 2002.

[11] Harary,F., Graph Theory, Addition Wesley, Third Printing, October 1972.

[12] Zimmermann, H.J., Fuzzy Set Theory and its Applications, Kluwer-Nijhoff, Boston, 1985.

[13] NagoorGani.AJahirHussain. R, and Yahya Mohamed. S ,Irregular Intuitionistic fuzzy graph, IOSR Journal Mathematics Vol. 9, Issue 6 (Jan. 2014), PP 47-51. 